\title{
Positional Change-Induced Arrhythmia during General Anesthesia Related to Caudal Movement of the Peripherally Inserted Central Venous Catheter: A Case Report
}

\author{
Isao Utsumi ${ }^{1}$, Tomasz Hascilowicz ${ }^{1}$, Yasushi Mio ${ }^{1}$, and Sachiko Omi ${ }^{1}$ \\ ${ }^{1}$ Tokyo Jikeikai Ika Daigaku Fuzoku Daisan Byoin
}

March 15, 2021

\begin{abstract}
We present a case of arrhythmia developed after positional change in a patient under general anesthesia, who had peripherally inserted central venous catheter (PICC) inserted by ECG-guided tip confirmation system. We stress the possibility of cardiac arrhythmias related to caudal movement of the PICC catheter upon changes in patient's position.
\end{abstract}

\section{INTRODUCTION}

Complications related to peripherally inserted central venous catheters (PICCs), such as arterial puncture, pneumothorax, hemothorax, and infections, are reported to occur less frequently than with other central venous catheters, ${ }^{1,2}$ and PICC use has increased in many clinical settings including the perioperative period. Consequently, more patients have been undergoing surgery under general anesthesia with PICCs inserted before operation. PICC usage has become more widespread also due to newly introduced and more convenient insertion techniques, such as those adopting passive magnet tracking and electrocardiogram (ECG)-guided tip confirmation system (TCS), ${ }^{3}$ that facilitates catheter placements at the bedside without confirmatory fluoroscopy and/or chest X-ray radiography and insertions performed by trained nurse practitioners. It has been well known that guidewires used during PICC placement may cause disturbances of cardiac rhythm when progressed too far into the right atrium (RA). ${ }^{4}$ There are limited case reports on positional changerelated arrhythmias due to too far insertion of PICCs in awake patients ${ }^{5,6}$; however, arrythmias that occurred during general anesthesia and were induced by a catheter inserted with the TCS-guided technique have not been reported.

We present a case of nonsustained ventricular tachycardia (VT) that developed upon change in patient's position under general anesthesia. Discussion on factors related to positional change-related arrhythmias in patients with preoperatively inserted PICCs using TCS is carried out and possible solutions are suggested.

A written consent was obtained from the patient for publication of this case report and the accompanying images in compliance with the hospital institutional review board regulations.

\section{CASE REPORT}

The patient was a 44-year-old woman (height $155 \mathrm{~cm}$, weight $62 \mathrm{~kg}$ ), scheduled for laparoscopic right adrenalectomy for primary aldosteronism. She had no previous history of arrhythmia and did not use any medications causing cardiac rhythm disturbances. On the day before surgery, a PICC (PowerPICC ${ }^{\circledR}$, Becton, Dickinson and Company, NJ, USA) was inserted via the right brachial-basilic vein and the Sherlock 3CG TCS (Becton, Dickinson and Company, NJ, USA) was used to confirm the correct position of the catheter tip. It was placed $41 \mathrm{~cm}$ from the insertion site based on the maximal P-wave on ECG observed after confirmation of the P-wave inversion. The correct position of the tip was then confirmed by chest radiography. 
After induction of anesthesia, when the patient was placed in left lateral decubitus position with adduction and then internal rotation of her right shoulder and flexion of the elbow, monomorphic nonsustained VT occurred (Fig. 2). With further, almost maximal, internal rotation of the shoulder the arrhythmia ceased and the surgery commenced as planned. Intraoperatively, the right atrial and right ventricular pressures were measured through one of the PICC (Fig. 3) lumens. They indicated respiration-related movements of the catheter tip into the right ventricle; however, no further disturbances in the cardiac rhythm were observed. When the patient was turned back to the supine position after operation, the arrhythmia did not occur, and the post-operative course was uneventful.

\section{DISCUSSION}

"The Practical Guidelines for Safe Central Venous Catheter (CVC) Placement and Management" issued by the Japanese Society of Anesthesiologists (JSA, 2017) recommend zone B, an area around the junction of the left and right innominate veins and the upper superior vena cava (SVC), as the optimal position of catheter tip following insertion; ${ }^{7,8}$ these guidelines pertain to all central venous catheters and do not particularly focus on adequate positions of PICC tips. The European Society for Clinical Nutrition and Metabolism (ESPEN) guidelines (2009) also pertain to all CVC, presumably also including PICCs, and accordingly, the adequate site for catheter placement is when "the tip is in the lower third of the SVC, at the atrio-caval junction (CAJ), or in the upper portion of the RA." ${ }^{9}$ Similar recommendations are presented in "Safe Vascular Access (2016)" (UK) where the lower SVC or the upper RA position is considered optimal; ${ }^{10}$ however, some American authors exclude the upper RA and limit the optimal position of the catheter tip to the lower SVC and vicinity of the CAJ. ${ }^{11}$ Hence, adequate placement of the catheter recommended in the JSA guidelines is slightly shorter ${ }^{3}$ those guidelines, however, have been established with the goal of reducing frequency of CVC-related complications (vessel or myocardium perforation, thrombosis, occlusion, catheter-related infections, pneumothorax, arrhythmias, etc.) and have been based on reported data where CVC tip positions were confirmed either with X-ray fluoroscopy or portable chest radiography.

With technological advancements of magnetic tracking and intra-cavity ECG, PICCs have been recently inserted at the bedside without X-ray fluoroscopy or post-insertional chest radiography. PICCs placed with the Sherlock 3CG TCS, where tip position at the CAJ is considered correct accordingly with the observed inversion of the electrocardiographic P-wave (NICE Medical Technology Guidance), tend to be inserted too far into the RA. Malposition of the catheter tip with the Sherlock 3CG does occur and reach proportions of $56.1 \%$ or $20.5 \%$, depending on the definition of the adequate tip position (low SVC/CAJ or mid SVC/low SVC/CAJ/high RA, respectively). ${ }^{12}$ According to Johnston et al.malposition might be due to specific characteristics of the 3CG technology (targeting the CAJ, which is sometimes impossible to achieve in clinical settings), difficulty in defining the CAJ position on chest radiographs and/or inconsistent CVC placement guidelines. ${ }^{12}$ Thus, even if the catheter is inserted and fixed in a suitable position, complications might still occur.

Arrhythmias related to PICCs are rare. In three reported cases, ${ }^{5,6}$ nonsustained VT occurred in awake patients upon change in their body position (usually from supine to lateral decubitus) following PICC placement and confirmation of the tip position in the lower SVC by fluoroscopy or chest radiography. In our case, PICC was inserted with the Sherlock 3CG TCS one day before surgery and arrhythmia did not occur until the patient was positioned for operation under general anesthesia. Movements of the catheter tip (caudally up to $5.3 \mathrm{~cm}$ [or 2.2 rib spaces]) ${ }^{13}{ }^{14}$ due to shoulder adduction have been reported, but only rarely do they induce ventricular tachyarrhythmias. The catheter tips constantly moving accordingly with the blood/injection fluid flow changes in the vicinity of the CAJ (lower risk of thrombosis ${ }^{12}$ ) or upper RA might occasionally contact the atrioventricular (AV) node/right ventricular wall; however, if this contact is only momentary, arrhythmia will presumably not occur. This might change in lateral decubitus or prone position when the contact with the AV node/ right ventricular wall ${ }^{6}$ would last longer and, thus, induce VT that is not observed immediately after PICC insertion (usually in supine position). A minor change in body position might terminate the arrhythmia (in our case, further adduction of the shoulder ) but respiration- and blood flow-related movements would not cease (Fig. 3). In an awake patient, arrhythmia 
might produce symptoms like dyspnea, chest pain, or palpitations and, thus, provoke a timely response ${ }^{5,6}$ contrary to that in patients being under general anesthesia, in whom suspicion and vigilant observation of the ECG tracing during changes in body position remain the only means of early recognition and treatment of PICC-induced arrhythmias. Simulation of the intraoperative position immediately after PICC insertion might allow detection of positional change-induced arrythmias and prompt early correction of the catheter position (usually pulling back the catheter); however, it is not always possible in clinical settings.

\section{CONCLUSION}

Notwithstanding inconsistencies in CVC placement guidelines, ${ }^{7,9,10}$ TCS-guided PICC insertions seem to produce lower rates of tip malposition and PICC-related complications; however, PICCs inserted with the Sherlock 3CG TCS tend to be located further into the RA or right ventricle (RV) than conventionally placed PICCs under fluoroscopic or radiographic guidance, and might induce arrhythmias upon changes in patient's position. In unconscious and sedated patients as well as in patients under general anesthesia, these arrhythmias should be suspected, and vigilant ECG monitoring should be performed. Positioning the patient in the final intraoperative position immediately after PICC insertion might be reasonable; however, further studies are required to assess the validity of its routine implementation.

\section{Conflict of Interest}

None

\section{Ethical Considerations}

A written consent was obtained from the patient for publication of this case report and the accompanying images in compliance with the hospital institutional review board regulations.

\section{Funding}

None

\section{Author Contributions:}

Isao Utsumi: This author provided clinical information about the patient's course and wrote and reviewed the manuscript.

Tomasz Hascilowicz: This author provided clinical information about the patient's course and contributed to the drafting of the manuscript.

Yasushi Mio: This author helped revise and approve the final draft of the manuscript.

Sachiko Omi: This author helped revise and approve the final draft of the manuscript.

\section{REFERENCES}

1. Amerasekera, S. S., Jones, C. M., Patel, R., Cleasby, M. J. Imaging of the complications of peripherally inserted central venous catheters. Clin Radiol . 2009;64(8):832-840.

2. Grau, D., Clarivet, B., Lotthe, A., Bommart, S., Parer, S. Complications with peripherally inserted central catheters (PICCs) used in hospitalized patients and outpatients: a prospective cohort study. Antimicrob Resist Infect Control . 2017;6:18.

3. Dale, M., Higgins, A., Carolan-Rees, G. Sherlock 3CG((r)) Tip Confirmation System for Placement of Peripherally Inserted Central Catheters: A NICE Medical Technology Guidance. Appl Health Econ Health Policy . 2016;14(1):41-49..

4. Lee, J. M., Lee, J., Hwang, J. Y., et al. Randomized comparison of three guidewire insertion depths on incidence of arrhythmia during central venous catheterization. Am J Emerg Med . 2017;35(5):743-748. 
5. Bivins, M. H., Callahan, M. J. Position-dependent ventricular tachycardia related to a peripherally inserted central catheter [published correction appears in Mayo Clin Proc 2000 May;75(5):542]. Mayo Clin Proc . 2000;75(4):414-416.

6. Alvarez, P., Schurmann, P., Smith, M., Valderrabano, M., Lin, C. H. Position-Dependent Ventricular Tachycardia Related to Peripherally Inserted Central Venous Catheter. Methodist Debakey Cardiovasc J . 2016;12(3):177-178.

7. Safety Committee of Japanese Society of Anesthesiologists. Practical guide for safe central venous catheterization and management 2017. J Anesth . 2020;34(2):167-186.

8. Stonelake, P. A., Bodenham, A. R. The carina as a radiological landmark for central venous catheter tip position. Br J Anaesth . 2006;96(3):335-340.

9. Pittiruti, M., Hamilton, H., Biffi, R., MacFie, J., Pertkiewicz, M. ESPEN. ESPEN Guidelines on Parenteral Nutrition: central venous catheters (access, care, diagnosis and therapy of complications). Clin Nutr . 2009;28(4):365-377.

10. Bodenham, C. A., Babu, S., Bennett, J., et al. Association of Anaesthetists of Great Britain and Ireland: Safe vascular access 2016 [published correction appears in Anaesthesia. 2016 Dec;71(12 ):1503]. Anaesthesia . 2016;71(5):573-585.

11. Infusion Nurses Society. Infusion Nursing Standards of Practice. J Infus Nurs . 2006;29(1 Suppl):S1-S92.

12. Johnston, A. J., Holder, A., Bishop, S. M., See, T. C., Streater, C. T. Evaluation of the Sherlock 3CG Tip Confirmation System on peripherally inserted central catheter malposition rates. Anaesthesia . 2014;69(12):1322-1330.

13. Forauer, A. R., Alonzo, M. Change in peripherally inserted central catheter tip position with abduction and adduction of the upper extremity. J Vasc Interv Radiol . 2000;11(10):1315-1318.

14. Connolly, B., Amaral, J., Walsh, S., et al. Influence of arm movement on central tip location of peripherally inserted central catheters (PICCs). Pediatric Radiology. 2006;36(8):845-850.

\section{FIGURE LEGENDS}

Figure 1. A confirmatory chest X-ray taken after insertion of the peripherally inserted central catheter (PICC).

Figure 2. An electrocardiogram (lead II) showing nonsustained ventricular tachycardia (VT) that occurred during positional change from supine to left lateral decubitus position with adduction and internal rotation of the right arm.

Figure 3. The right atrial and right ventricular pressures measured through one of the PICC lumens during operation. (HR: heart rate, ART: arterial pressure, CVP: central venous pressure) 
Figure. 1

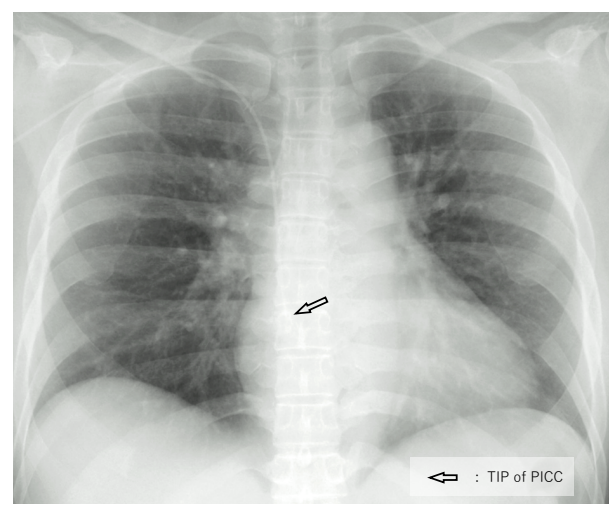

Figure. 2

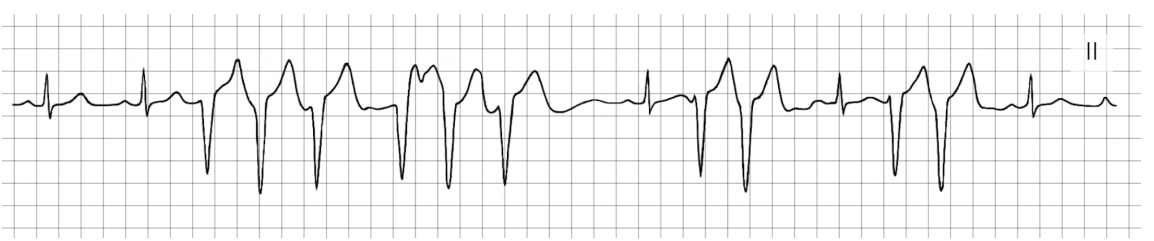

Figure. 3

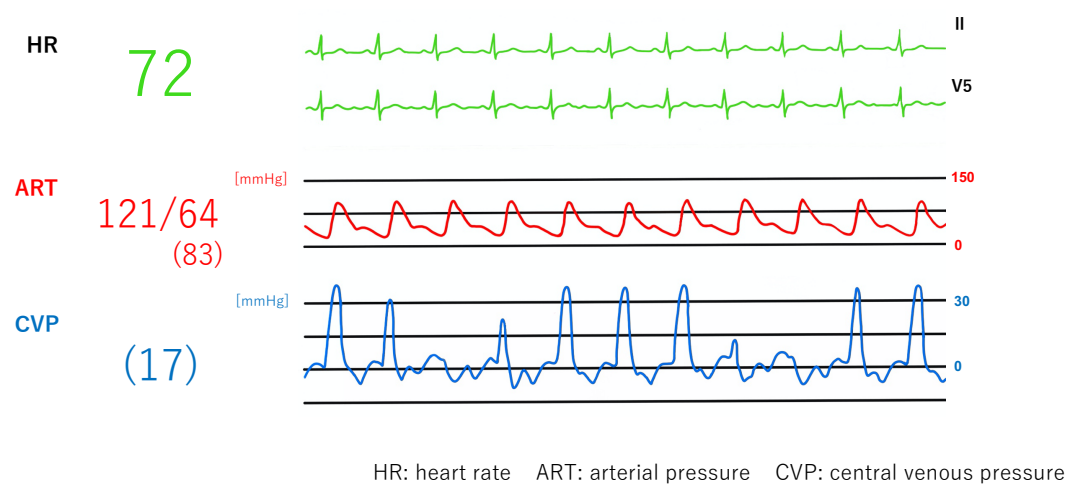

